\title{
Analysis of Clinicopathologic Characteristics of Gastric Cancer
}

\author{
Zhao Xueyu, Li Donghai, Liu Ming \\ Department of General Surgery, Affiliated Hospital of Inner Mongolia Medical University, Huhhot, P. R. China
}

Email address:

liumingfy@163.com (Liu Ming)

\section{To cite this article:}

Zhao Xueyu, Li Donghai, Liu Ming. Analysis of Clinicopathologic Characteristics of Gastric Cancer. Cancer Research Journal. Vol. 6, No. 3, 2018, pp. 79-83. doi: 10.11648/j.crj.20180603.12

Received: May 23, 2018; Accepted: June 7, 2018; Published: June 29, 2018

\begin{abstract}
Objective Western Inner Mongolia is a high risk area of gastric cancer. However, detailed clinical and pathological data of gastric cancer has been lacking. Our hospital is one of the largest gastric cancer diagnoses and treatment centers in western Inner Mongolia. This study was designed to investigate the characteristics of clinical data of patients with gastric cancer in Western Inner Mongolia at our hospital over the past eight years. Methods Data of 1548 cases of gastric cancer patients from 2007.01.01 to 2015.01.01 in our hospital was retrospectively analyzed for their clinical characteristics. Results Of the 1548 patients, 1251 cases $(80.81 \%)$ were male and $297(19.19 \%)$ were female, and the average age was $60.94 \pm 10.90$ years old. The cancer location was mainly on the antrum, accounting for $40.76 \%$. Different ages of gastric cancer location constituent ratio was different, and the difference was significant $\left(\chi^{2}=39.836, \mathrm{P}=0.000\right)$. Poorly differentiated adenocarcinoma was a major histological type, accounting for $46.58 \%$. Different ages of histological classification and the differentiation constituent ratio was also different, and the difference was significant $\left(\chi^{2}=67.300, \mathrm{P}=0.000\right)$. There were 144 cases of early gastric cancer, accounting for $9.30 \%$; 1404 cases of advanced gastric cancer, accounting for $90.70 \%$. The majority pathologic type of advanced gastric cancers were Bormann III(53.92\%). III+IV stage was a majority of TNM stage, accounting for $72.41 \%$. Different ages of TNM stage was different, the difference was significant $\left(\chi^{2}=9.013, \mathrm{P}=0.029\right)$. Conclusion Advanced gastric cancer is the major type of patients with gastric cancer in Western Inner Mongolia. Gastric cancer has a higher incidence in older male patients. Poorly differentiated adenocarcinoma and cancer in antrum have a larger probability. Compared with older patients, there is higher occurrence of malignant cancer and earlier TNM stage in younger patients. Mongolian patients and Han patients showed no significant difference.
\end{abstract}

Keywords: Gastric Cancer, Data Base, Clinic Pathologic Characteristic, Western Inner Mongolia

\section{Introduction}

Gastric cancer is the $4^{\text {th }}$ commonest cancer in the world. It is also the $2^{\text {nd }}$ deadliest malignant tumor. World widely, more than $70 \%$ of new cases of gastric cancer and deaths occur in developing countries, while the highest incidence is in the East of Asia. [1] Japan, Korea and China all have high incidence of gastric cancer. There are about 400,000 new cases each year in China, accounting for $42 \%$ [2-3] of the total number of cases in the world. According to statistics, the incidence rate of gastric cancer in China was $36.21 / 100,000$, mortality rate is 25.88/100,000, which are both higher than the world average level. [3] Located in northern China, Inner Mongolia is an autonomous region with a high incidence of gastric cancer. Its economy, medical conditions and other conditions are relatively backward. Our hospital is the largest center of diagnosis and treatment for gastric cancer in the western part of Inner Mongolia. A database of clinical and pathological data of octal gastric cancer was analyzed to understand the characteristics of gastric carcinoma in the area, and provide a basis for prevention and treatment of gastric cancer.

\section{Materials and Methods}

\subsection{Research Object}

1548 cases were selected from January 01, 2007 to January 01,2015 in the database of gastric carcinoma patients in our hospital. All cases were clearly diagnosed. And their clinical and pathological information is complete. 


\subsection{Diagnosis and Staging}

\subsubsection{Pathological Diagnosis}

Histopathology in accordance with WHO (2000) classification standard divides the gastric cancer into adenocarcinoma, papillary adenocarcinoma, tubular adenocarcinoma, Mucinous Carcinoma, Signet-Ring cell carcinoma and other types; and according to the degree of cell differentiation, into high, medium and low levels of differentiations.

\subsubsection{TNM Staging}

Using the 7th Edition AJCC TNM staging standard of gastric cancer in 2010, with pre-surgery examinations, intraoperative and postoperative pathological findings to determine depth of tumor invasion $(\mathrm{T})$, violations of lymph nodes (N) and metastasis (M) stage 0, I, II, III, IV.

\subsubsection{Tumor Locations}

Tumor location sites are divided into the gastric antrum (sinus lesser curvature of stomach, Big Bend and the angle of the stomach), body of stomach, Cardia and fundus of stomach, stomach (including extensive involvement of 2 or more locations) and stomach stump.

\subsection{Statistical Methods}

Statistical analysis software SPSS 17.0 will be used. Statistics for count data, using $\chi^{2}$ test, test level $\alpha=0.05$.

\section{Results}

\subsection{Comparison of Changes of 2007-2014 Gastric Cancer Patients}

The number of diagnosis and treatment for gastric cancer in those 8 years showed an increasing trend. In years 09-10 show the biggest increase. Fixed base ratio increased by $32.2 \%$; link relative ratio increased by $32.2 \%$. As shown in table 1 .

Table 1. Comparison of treatment of patients with gastric cancer in 2007-2014.

\begin{tabular}{llllllll}
\hline \multirow{2}{*}{ Year } & \multirow{2}{*}{ Cases } & \multicolumn{2}{l}{ Absolute growth (cases) } & Development speed (\%) & Growth rate (\%) \\
\cline { 3 - 7 } & Total & Year by year & Relative ratio with fixed base & Link Relative ratio & Relative ratio with fixed base & link Relative ratio \\
\hline $07-08$ & 273 & - & - & 100 & - & - \\
$09-10$ & 361 & 88 & 88 & 132.2 & 132.2 & 32.2 \\
$11-12$ & 420 & 147 & 59 & 153.8 & 116.3 & 53.8 \\
$13-14$ & 494 & 221 & 74 & 181.0 & 117.6 & 81.0 \\
\hline
\end{tabular}

\subsection{Gender and Age Distribution}

Among the 1548 cases, 1251 were male (80.81\%) and 297 were female (19.19\%). The gender ratio of male: female is $4.21: 1$. Patients aged 25-91 years, median age of 61 years old. Average age is $60.94 \pm 10.90$. Gender composition of patients of all ages is shown in table 2 .

Table 2. 1548 cases of gastric cancer with age and gender composition.

\begin{tabular}{|c|c|c|c|c|c|}
\hline \multirow{2}{*}{ Gender } & \multicolumn{4}{|l|}{ Year } & \multirow{2}{*}{ Total } \\
\hline & $<45(\%)$ & 45-59 (\%) & $60-74(\%)$ & $>74(\%)$ & \\
\hline male & $87(6.95)$ & $471(37.65)$ & $597(47.72)$ & $96(7.67)$ & $1251(100)$ \\
\hline female & $56(18.86)$ & $107(36.03)$ & $112(37.71)$ & $22(7.41)$ & $297(100)$ \\
\hline total & $143(9.24)$ & $578(37.34)$ & $709(45.80)$ & $118(7.62)$ & $1548(100)$ \\
\hline M-F & $1.55: 1$ & $4.40: 1$ & 5.33:1 & $4.36: 1$ & 4.21:1 \\
\hline
\end{tabular}

\subsection{Pathological Features}

\subsubsection{Tumor Locations}

The data shows in gastric carcinoma 631 cases $(40.76 \%)$ in gastric antrum; 414 cases $(26.74 \%)$ in the body of the stomach; $467(30.17 \%)$ cases in cardiac and gastric fundus, 12 cases of total gastrectomy $(0.78 \%)$, and 24 cases $(1.55 \%)$ in stomach stump.

With the analysis of cancer sites grouped by ages, it was found that gastric lesion in different age ratio is different, with a statistically significant difference $\left(x^{2}=39.836\right.$, $\mathrm{P}=0.000$ ). Cancer of the Cardia and stomach fundus ratio gradually increases with age, from the $18.18 \%$ of the < 45 -year age group to the $36.44 \%$ of the $>74$-year age group. In both groups there was a significant difference $\left(\mathrm{x}^{2}=11.082\right.$, $\mathrm{P}=0.001)$. Gastric cancer as the location has the highest percentage of all ages. As shown in table 3.

Table 3. Comparison of lesion of gastric cancer in different age.

\begin{tabular}{lllllll}
\hline Location & $<\mathbf{4 5}(\mathbf{\%})$ & $\mathbf{4 5 - 5 9 ( \% )}$ & $\mathbf{6 0 - 7 4}(\mathbf{\%})$ & $\mathbf{> 7 4 ( \% )}$ & $\mathbf{P}$ \\
\hline Fundus and Cardiac & $26(18.18)$ & $143(24.74)$ & $255(35.97)$ & $43(36.44)$ & 31.352 \\
gastricum & $52(36.36)$ & $163(28.20)$ & $179(25.25)$ & $20(16.95)$ & 13.970 \\
antrum & $62(43.36)$ & $258(44.64)$ & $259(36.53)$ & $52(44.07)$ & 9.784 \\
stomach & $1(0.70)$ & $4(0.69)$ & $5(0.71)$ & $2(1.69)$ & - & 0.003 \\
gastric stump & $2(1.40)$ & $10(1.73)$ & $11(1.55)$ & $1(0.85)$ & - \\
\hline
\end{tabular}




\subsubsection{Histological Types and Differentiation}

In the 1548 cases of gastric cancer, regarding the tissues of patients, most had low differentiated adenocarcinoma with 721 cases, accounting for $46.58 \%$. This was followed by a moderately differentiated adenocarcinoma of 451 cases, accounting for $29.13 \%$ of the total. 112 cases had malignant with higher levels of Signet-Ring cell carcinoma, accounting for $7.24 \%$. Other organization types include squamous cell carcinoma and adenosquamous carcinoma and carcinoid etc.
By age group and tissue type, gastric cancer tissue types vary in different age groups, there was a significant difference $\left(\mathrm{x}^{2}=67.300, \mathrm{P}=0.000\right)$ in $<45$-year group. Compared with other groups of poorly differentiated adenocarcinoma ratio is high, there was a significant difference $\left(\mathrm{x}^{2}=8.030, \mathrm{P}=0.045\right)$, Signet-Ring cell carcinoma constitute a relatively high portion compared with other groups, there was a significant difference $\left(x^{2}=24.793\right.$, $\mathrm{P}=0.000$ ), see table 4 .

Table 4. Type of gastric cancer patients and the degree of differentiation.

\begin{tabular}{|c|c|c|c|c|c|c|}
\hline $\begin{array}{l}\text { Histological type } \\
\text { Adenocarcinoma }\end{array}$ & $<45(\%)$ & $45-59(\%)$ & $60-74(\%)$ & $>74(\%)$ & $\chi^{2}$ & $\mathbf{P}$ \\
\hline poorly differentiated & $80(55.94)$ & $278(48.10)$ & $310(43.72)$ & $53(44.92)$ & 8.030 & 0.045 \\
\hline moderately differentiated & $16(11.19)$ & $149(25.78)$ & $248(34.98)$ & $38(32.20)$ & 37.726 & 0.000 \\
\hline well differentiated & $3(2.10)$ & $21(3.63)$ & $37(5.22)$ & $10(8.47)$ & 7.948 & 0.047 \\
\hline signet ring cell cancer & $24(16.78)$ & $45(7.79)$ & $37(5.22)$ & $6(5.08)$ & 24.793 & 0.000 \\
\hline mucinous adenocarcinoma & $17(11.89)$ & $71(12.28)$ & $64(9.03)$ & $8(6.78)$ & 5.659 & 0.129 \\
\hline other & $3(2.10)$ & $14(2.42)$ & $13(1.83)$ & $3(2.54)$ & - & - \\
\hline
\end{tabular}

\subsubsection{Staging and Bormann Type}

In the 1548 cases of gastric cancer, 144 cases were early gastric cancer (9.3\%), 1404 were advanced gastric cancer (90.7\%). Most cases were Bormann type III up advanced gastric cancer with 757 cases, accounting for $53.92 \%$; the rest is I-183, 13. 3\%; 394 cases of type II , 28.06\%; and type IV $70,4.99 \%$.

Using TNM staging of gastric cancer, T stage T4 had 662 patients. In N staging, 653 cases were $\mathrm{N} 1$ stage, accounted for $42.18 \%$. In M staging, 1201 were in MO stage, which accounted for $77.58 \%$. Staging in patients with early gastric cancer $0+$ primary + secondary period reached a total of 427 cases of $27.58 \%$. Patients in + late stage III and IV gastric cancer was the majority, with a total of 1121 , accounting for $72.41 \%$. According to the age groups, in TNM staging the $<45$ age group had more cases in earlier stages than in other groups. There was a significant difference $\left(x^{2}=9.013\right.$, $\mathrm{P}=0.029$ ), see table 5 .

Table 5. Comparison of TNM staging of gastric cancer patients.

\begin{tabular}{lllll}
\hline Staging & $<\mathbf{4 5}(\mathbf{\%})$ & $\mathbf{4 5 - 5 9 ( \% )}$ & $\mathbf{6 0 - 7 4}(\%)$ & $>\mathbf{7 4}(\mathbf{\%})$ \\
\hline 0+I+II & $54(37.76)$ & $161(27.85)$ & $182(25.67)$ & $30(25.42)$ \\
III+IV & $89(62.24)$ & $417(72.15)$ & $527(74.33)$ & $88(74.58)$ \\
\hline
\end{tabular}

\subsection{Comparison Between Han and Mongolian}

In the 1548 patients, there were 1440 Han patients, accounting for $93.02 \%(1440 / 1548) .108$ patients were Mongolian, accounting for $6.98 \%$ (108/1548). The number of Mongolian patients was relatively small. The ethnic group comparison of relevant clinical information found no statistically significant differences between the Han and Mongolian patients.

\section{Discussion}

Western region of Inner Mongolia is an area of high incidence of gastric cancer in China. However, with such high incidence rate, detailed clinical and pathological information report had been lacking. Our hospital is located in the western part of Inner Mongolia, and is the region's largest centre for diagnosis and treatment of gastric cancer. A gastric cancer database in the hospital was built in 2007 . Analyzing the database can indirectly reflect the characteristics of gastric carcinoma in Western Inner Mongolia, to provide the scientific basis for decision-making in prevention and treatment of gastric cancer in the region.

\subsection{Patient Trends and General Information}

This research shows that in the past 8 years in our hospital the number of patients with gastric cancer increased year by year. This may be related to economic improvement in Western Inner Mongolia, and the launch of early gastric cancer screening. According to the different regions of the country reported in various studies, [4-11] the average age of patients with gastric cancer is 55.70-64.89 years. This research shows a similar result. Jiangsu, Henan, Hunan, Guangdong, Beijing, and other regions have shown, male and female gender ratio was approximately (1.84-3.04): 1 . This was close to the WHO released [12] ratio of the incidence rate of gastric cancer in men and women worldwide 2.32:1. Similar with studies about Xinjiang and Shanxi, in this group there was a bigger proportion of male patients; the gender ratio is 3.36:1, 3.86:1. Life habits in different regions also has an effect, that the higher proportion of male patients may be connected with their smoking, drinking, preference of salted and smoked food over fresh fruits and vegetable. [13-14]

\subsection{Tumor Locations}

Foreign researches in recent years [12] showed a decreased incidence of distal gastric cancer, and the increase of Cardia and fundus stomach cancer, which became the most incidence site. Smoking, drinking, obesity, reflux are risk factors for Cardia cancer. Meanwhile, the popularity of Helicobacter pylori eradication treatment contributed to the 
controlling of gastric cancer [15] and the relative increase in the incidence of cardiac carcinoma cancer. Many Chinese researches showed that, [5-11] incidence sites for gastric cancer differ in different regions. Cardia fundus and antrum now have the highest occurence in China.

Through this set of data it can be seen among patients with gastric cancer, gastric distal gastric cancer still occurs the most in this region. This was followed by proximal stomach. Compared with older patients, incidence site of young people is lower. With the increase of age, under the lesion of gastric cancer, location to the proximal stomach increases. According to research, [16] this was mainly because of the moving on of the fundic glands atrophy, junction, increased intestinal metaplasia, predilection to move high gastric cancer as the patients' age increased. In addition, the lower esophageal sphincter tension in the elderly is poorer, causing gastroesophageal reflux; plus the influence of physical and chemical stimuli such as eating, causing inflammation, repairing, hyperplasia, carcinoma of gastric Cardia and a series of pathological changes.

\subsection{Pathologic Types}

Most histological types were poorly differentiated adenocarcinoma. This is generally in consistence with the majority of researches in China. [4-11] Stomach carcinoma in the young tissue-typing is bad. Poorly differentiated adenocarcinoma and Signet Ring cell cancer is higher than the other age groups. Moderately differentiated adenocarcinoma is also higher than the other group, which is also in consistence with earlier studies. [5-9, 17] It is possible that younger patients may themselves be genetically susceptible. As researches has found that hereditary diffuse type gastric cancer more $<45$ age group, its onset is based on coding of epithelial type calcium sticky protein of CDH1 gene embryo Department mutation. Based on this experience, including promoter methylation, somatic mutation and loss of heterozygosity, including a "two-hit", led to wild-type CDH1 allelic loss of function that caused the disease; while later onset are more likely to be the result of long-term effects of external factors.

\subsection{Bormann and Staging}

Currently the early gastric cancer detection rate is about $9.6 \%-13.2 \%$; [18] this is much lower compared with Korea, and Japan and other countries. In Japan, early gastric carcinoma in gastric cancer has accounted for more than $50 \%$ of their total cases [3]. In the data used by this paper, early gastric cancer accounted for $9.3 \%$ of the total, which only reaches the national low level. The majority are advanced gastric cancer. Most cases are Bormann type III or above advanced gastric, followed by type II. From the perspective of TNM staging of gastric cancer, most patients are at relatively late stages, and the prognosis is poor. Under age stratification was found that young people are in relatively earlier TNM staging. This may be related to the improvement of young people's health consciousness in recent years.
Stomach cancer is a disease with high mortality rates. Early diagnosis and treatment is important. Promoting the gastric cancer screening and crowd of gastric cancer awareness in this area will help to improve the early detection of gastric cancer in the region.

\subsection{Ethnic Background}

Inner Mongolia autonomous region in which Han Chinese is the major population; while Mongolian is the major minority group. This study found no significant differences in clinical and pathological features between Han and Mongolian people. Still Mongolian patients with gastric cancer needs further study.

\section{Conclusion}

To sum up, by reviewing the information on diagnosis and treatment of patients with gastric cancer in the past 8 years in our hospital, clinical and pathological characteristics of gastric cancer in this region was studied. This can be referred to make the next step of gastric cancer prevention programs, improve the detection of early gastric cancer and ultimately reduce the mortality rate and improve the quality of life. However, this study has certain limitations, including a short time span of study, small sample sizes, especially that of Mongolian patients. Also long term surviving patients were not included in the study, which require further additional researches.

\section{References}

[1] Torre LA, Bray F, Siegel RL, et al. Global cancer statistics, 2012 [J]. A Cancer Journal for Clinicians, 2015, 65 (2):87-108.

[2] Zou Wenbin, Li Zhao-Shen. Research progress of gastric cancer incidence and mortality in China $[\mathrm{J}]$. Chinese Journal of practical internal medicine, 2014, 34 (4): 408-415.

[3] Park J Y, Von K L, Herrero R. Prevention Strategies for Gastric Cancer: A Global Perspective[J]. Clinical Endoscopy, 2014, 47 (6):478-489.

[4] Xu Xiaoqin, Zhang Wenli, Han Cunzhi, et al. from 2001 to 2010, Shanxi provincial Cancer Hospital: characteristic analysis of inpatients with gastric cancer [J]. Cancer research and clinical, 2015, 27 (6): 413-417.

[5] Xie Honghu, Lv Chengyu, Chen Wei, et al. Clinical pathological analysis of 958 cases [J]. Chinese Journal of general basic and clinical, 2011, 18 (2): 153-158.

[6] Zhao Jinpeng, Feng Jin, Xie Haibin, et al. 2338 cases analysis of clinical and pathological characteristics in patients with gastric cancer surgery [J]. Jiangsu pharmaceutical, 2015, 41 (1): 41-44.

[7] Jing Xiao Yue, Wang Jianguo, Zhou Bing, etc. nearly 10 years clinical epidemiological characteristics of gastric cancer in Henan Province [J]. Chinese Journal of general basic and clinical, 2010, 17 (1): 29-33. 
[8] Han, Luo Yimin, Ivan. Clinical epidemiological characteristics and analysis of gastric carcinoma in 1539 cases [J]. Chinese Journal of clinical physician: Electronic Edition, 2015, 9 (5): 61-64.

[9] Zhang Baoyan, Li Xianghong, Yuan Jing, et al. 694 cases analysis of clinical and pathological characteristics of gastric cancer [J]. Chinese Journal of digestive tract, 2013, 33 (1): 33-36.

[10] mayu flowers. Characteristic analysis of 424 cases of gastric cancer in different nationalities in Xinjiang [J]. Modern cancer medicine, 2012, 20 (9): 1891-1893.

[11] Liu Guanhong, He Haiping, Chen Jiansi. Cancer Hospital for nearly 10 years and clinical epidemiological analysis $[\mathrm{J}]$. Modern cancer medicine, 2013, 21 (12): 2835-2838.

[12] BERNARD W. STEWART, et al. WORLD CANCER REPORT 2014 [M]. International Agency for Research on Cancer, 2014:56-58.

[13] Cen Chao, Wang Chao, Zhou Xihan, et al. case-control study on risk factors for stomach cancer in Baise [J]. Chinese
Journal of cancer prevention and control, 2014, 21 (3): 178-182.

[14] Woo H D, Park S, Oh K, et al. Diet and cancer risk in the Korean population: a meta-analysis [J]. Asian Pacific Journal of Cancer Prevention Apjcp, 2014, 15 (19):8509-8519.

[15] Li Yixin, Li Xiuming, Zhang nan, et al. Helicobacter pylori infection and gastric cancer development and its correlation with prognosis $[\mathrm{J}]$. Chinese Journal of cancer prevention and control in 2015, 22 (2): 92-94.

[16] Zhou Sining, Zhou Xiaolan. Different parts of clinical and Pathologic feature and biological behavior of gastric cancer tissue [J]. World Chinese Journal of Digestology, 2014, 22 (18): 2607-2611.

[17] Isobe T, Hashimoto K, Kizaki J, et al. Characteristics and prognosis of gastric cancer in young patients [J]. Oncology Reports, 2013, 30 (1):43-49.

[18] Wei Wu, Wu Yunlin, Sun Ping, and so on. Evolution and experience of early gastric cancer rates [J]. Journal of Gastroenterology and hepatology, 2008, 17 (3): 205-208. 